\title{
Pulmonary adenocarcinoma in a slaughtered female Hanwoo
}

\author{
Hyoung-Seok Yang', Jae-Hoon Kim ${ }^{2, *}$ \\ ${ }^{1}$ Jeju Self-Governing Provincial Veterinary Research Institute, Jeju 63344, Korea \\ ${ }^{2}$ College of Veterinary Medicine, Veterinary Medical Research Institute, Jeju National University, Jeju 63243, Korea
}

\begin{abstract}
Pulmonary masses were observed in the thoracic cavity of a 9-year-old female Korean native cattle (Hanwoo) encountered at a Jeju, Korea slaughterhouse. Grossly, the thoracic cavity was filled with pulmonary masses. Histologically, the neoplastic masses were composed of many abnormal papillary structures lined by single to double columnar neoplastic cells and formed arborizing fronds supported by significant fibrovascular stroma. The neoplastic cells showed strong destructive and infiltrative growth to adjacent tissues and were strongly positive for pan-cytokeratin (CK), CK 7 and sporadically positive for CK MNF116. The masses in the female Hanwoo were diagnosed as a papillary pattern of pulmonary adenocarcinoma.
\end{abstract}

Keywords: adenocarcinoma, cattle, lung, papillary, slaughterhouse

\section{*Corresponding author}

Jae-Hoon Kim

College of Veterinary Medicine, Veterinary

Medical Research Institute, Jeju National

University, 102 Jejudaehak-ro, Jeju 63243,

Korea

Tel: $+82-64-754-3387$

Fax: +82-64-702-9920

E-mail: kimjhoon@jejunu.ac.kr

ORCID:

Hyoung-Seok Yang

https://orcid.org/0000-0003-4500-8826

Jae-Hoon Kim

https://orcid.org/0000-0002-4410-9126

Conflict of Interest

The authors declare no conflicts of interest.

Received: December 27, 2018

Revised: March 6, 2019

Accepted: March 11, 2019
Primary lung carcinomas of domestic animals are classified into adenocarcinoma, bronchioloalveolar carcinoma, squamous cell carcinoma, adenosquamous carcinoma, combined carcinoma, large cell carcinoma, small cell carcinoma, and ovine retroviral pulmonary carcinoma [1]. Pulmonary adenocarcinoma (PA) represents by far the most frequent type of primary pulmonary tumor in domestic animals [2]. PAs are generally very malignant, and intrapulmonary metastases in several lobes, as well as in bronchial lymph nodes, pleura, and even the brain, are not uncommon. Clinically, these neoplasms are associated with coughing, dyspnea, and sometimes fever due to secondary infections. In previous reports, histological features of primary epithelial lung tumors were reported for cattle in a British and Denver abattoir survey [3, 4]. In Korea, there have been reports of PA in dogs [5, 6], but not in other domestic animals including cattle. Herein, we report a case of PA of a slaughtered female Korean native cattle (Hanwoo) in Jeju.

Pulmonary masses distributed throughout all lung lobes were observed in the thoracic cavity of a 9-year-old female Hanwoo encountered at a Jeju slaughterhouse. The cow exhibited severe coughing, dyspnea, and extreme emaciation for several months before slaughter. Pulmonary mass samples were fixed in $10 \%$ neutral buffered formalin and embedded in paraffin wax, sectioned at $3 \mu \mathrm{m}$, and stained with hematoxylin and eosin (H\&E) for histological examination. In addition, Ziehl-Neelsen $(\mathrm{ZN})$ stain was used to distinguish bovine tuberculosis. Additional paraffin-embedded sections were maintained for immunohistochemistry (IHC) assessment. After mounting on silane-coated glass slides, each pulmonary mass section was stained by using a labeled streptavidin-biotin peroxidase method. To clarify the origin of the tumor cells, primary antibodies such as pan-cytokeratin (CK) clone AE1/AE3 (Dako, Denmark), CK clone MNF116 (Dako), CK 7 (Dako), vimentin (Dako), and chromogranin A (Dako) were used.

Grossly, the thoracic cavity was filled with massive pulmonary masses. The masses adhered to the thoracic pleural wall and pericardium (Fig. 1A). The pulmonary masses were variably sized, present in all lung lobes, yellowish in color, moderately firm, and rugged (Fig. 1B). Histologically, the neoplastic masses were composed of many abnormal papillary structures (Fig. 2A and B). These papillary structures were lined by single to double columnar neoplastic cells and formed arborizing fronds supported by significant fibrovascu- 

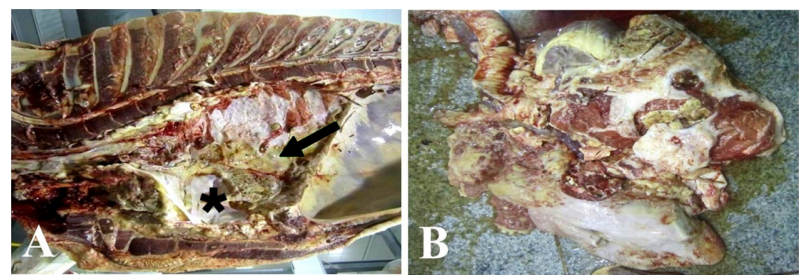

Fig. 1. Thoracic cavity and lung. (A) The pulmonary masses (arrow) adhered to the thoracic wall and pericardium $(*)$. (B) The pulmonary masses were yellowish in color, moderately firm, and rugged.
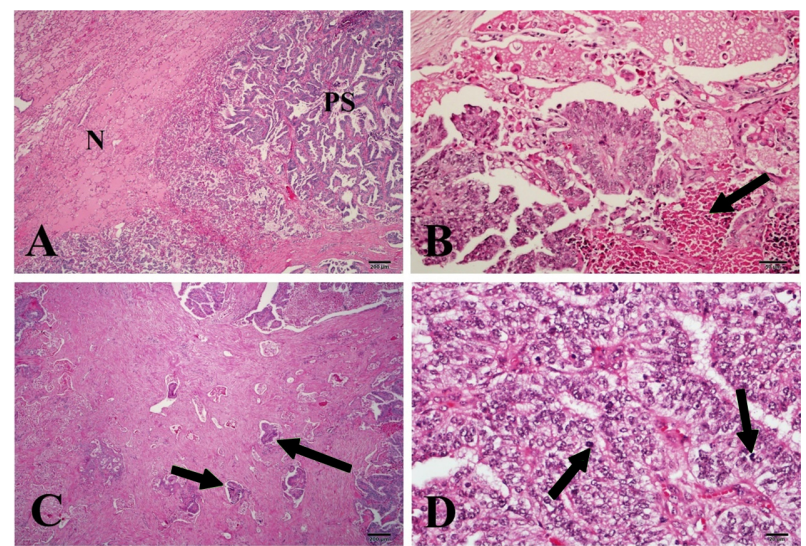

Fig. 2. Pulmonary masses. (A) Neoplastic masses were composed of papillary structures (PS) and showed strong destructive and infiltrative growth to normal tissue $(\mathrm{N})(\mathrm{H} \& \mathrm{E}$ stain; scale bar $=200 \mu \mathrm{m}$ ). (B) The papillary structures disintegrated under varying degrees of necrosis (arrow), leaving a mass of cellular or amorphous debris with inflammatory cells (H\&E stain; scale bar $=50 \mu \mathrm{m}) .(\mathrm{C})$ Note many neoplastic cell emboli (arrows) in blood vessels and lymphatics (H\&E stain; scale bar $=200 \mu \mathrm{m}$ ). (D) The epithelial cells lining the papillary structures were columnar with large ovoid basal nuclei and secreted mucus. Note mitotic figures (arrows) (H\&E stain; scale bar $=20 \mu \mathrm{m}$ ). $\mathrm{H} \& \mathrm{E}$, hematoxylin and eosin.

lar stroma. The papillary structures disintegrated under varying degrees of necrosis, leaving a mass of cellular or amorphous debris with inflammatory cells (Fig. 2B). These neoplastic epithelial cells showed strong destructive and infiltrative growth to adjacent tissues (Fig. 2A). Many neoplastic cells emboli were located in lymphatics and occasionally in blood vessels (Fig. 2C). The number of neoplastic cell mitotic figures $(0-1$ per high power field, on average) varied in different areas. The columnar epithelial cells lining the papillary structures had large ovoid basal nuclei and sometimes secreted mucus (Fig. 2D). The result of $\mathrm{ZN}$ staining for acidfast bacilli was negative in the pulmonary masses. The neoplastic cells were strongly positive for pan-CK (Fig. 3A), CK 7 (Fig. 3B and C), sporadically positive for CK MNF116 (Fig. 3D), and negative for vimentin and chromogranin A.

Based on the clinical and gross findings, as well as histopathologic and immunohistochemical results, the masses in

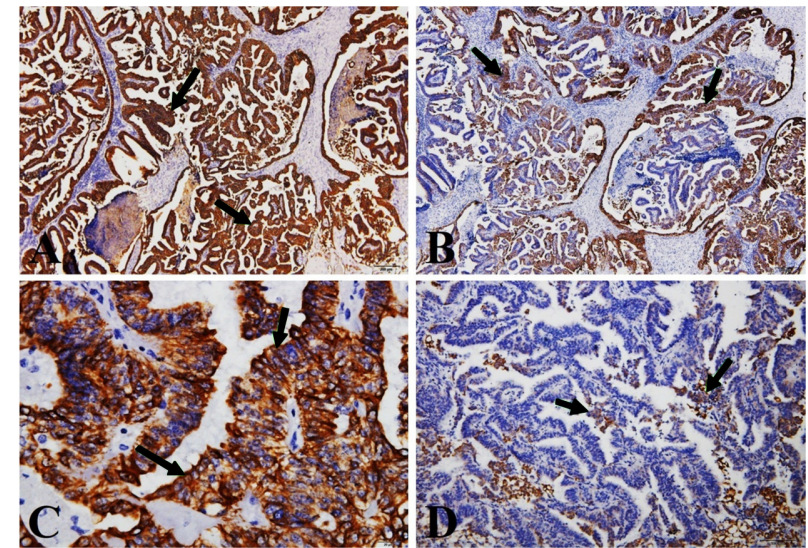

Fig. 3. Immunohistochemical results for CKs in pulmonary masses. (A) Neoplastic cells show strongly positive reactions (arrows) for CK AE1/AE3 antigens (IHC stain; scale bar $=200$ $\mu \mathrm{m})$. (B) Numerous CK 7 antigens present in papillary structures (IHC stain; scale bar $=200 \mu \mathrm{m}$ ). (C) High magnification image of (B). Note the strongly positive reactions (arrows) (IHC stain; scale bar $=20 \mu \mathrm{m}$ ). (D) Neoplastic cells were sporadically positive (arrows) for CK clone MNF116 (IHC stain; scale bar $=100 \mu \mathrm{m})$. CK, cytokeratin; IHC, immunohistochemistry.

the female Hanwoo in this study were diagnosed as pulmonary papillary adenocarcinoma.

Lung cancer is the most common major cancer type and cause of human mortality worldwide [7]. However, primary pulmonary neoplasia is an infrequent finding in domestic animals with the exception of dogs and cats [8]. Primary pulmonary tumors appear to be quite rare in cattle with surveys at slaughter indicating an incidence ranging between 2 and 20 cases per million cattle surveyed [3, 4, 8].

The most frequent clinical sign associated with pulmonary tumors in dogs is reported to be coughing (52\%), followed by dyspnea $(24 \%)$, lethargy (18\%), weight loss (12\%), anorexia, occasional vomiting, and paraneoplastic syndromes including hypertrophic osteopathy and hypercalcemia [8,9]. Similar to canine cases, the female Hanwoo with PA showed severe coughing, dyspnea, and extreme emaciation for several months.

In cattle, the usual metastatic sites of lung cancers are the bronchomediastinal lymph nodes [3], but metastasis frequently involves other nodes in the thorax and abdomen, such as pleura, liver, kidneys, and adrenals. According to a British abattoir survey, the detected lung cancers were all from female cattle, the majority of which were over 5 years old [3]. Unfortunately, we could not perform a histopathologic examination of regional lymph nodes of the thoracic cavity in this case. However, there was a possibility of metastasis of the PA due to the observation of very strong infiltrative growth of pulmonary neoplastic cells. In the present case, the cow with PA was 9-year-old, which is in agreement with observations in previous reports [3].

Further to the classifications of lung cancer published by 
the World Health Organization in 2004, a new classification strategy, based on a multidisciplinary approach to the diagnosis of lung adenocarcinoma, was published in 2011 [7]. According to the latter document, epithelial pulmonary tumors were reclassified into atypical alveolar hyperplasia, bronchioloalveolar adenoma (adenocarcinoma in situ), minimally invasive adenocarcinoma, adenocarcinoma, combined carcinoma, neuroendocrine or carcinoid tumor, and pulmonary blastoma [7, 8]. Bronchioloalveolar carcinoma was not included in the 2011 classification of lung tumor. The major histopathologic patterns of invasive PA have been classified into 5 categories: lepidic, papillary, micropapillary, acinar, and solid predominant with mucin. The lepidic growth pattern has tumor cells that appear to replace type II pneumocytes and Clara cells along the surface on alveolar walls. The lepidic tumor pattern is the classic bronchioloalveolar tumor pattern, which is composed of cuboidal or mildly flattened epithelial monolayer on alveolar-like structures [8]. Acinar adenocarcinoma presents as malignant glands invading a fibrous stroma. Papillary adenocarcinoma consists of malignant cuboidal to columnar tumor cells growing along fibrovascular cores in a papillary configuration, and the tumor cells in papillary form are more columnar than those in the lining cells of the lepidic form. The micropapillary growth pattern shows small papillary clusters of glandular cells growing within airspaces, and most clusters did not show fibrovascular cores. The solid adenocarcinoma with mucin pattern consists of sheets of tumor cells with abundant cytoplasm and mostly vesicular nuclei with several conspicuous nucleoli. Invasive mucinous adenocarcinoma presents as columnar cells filled with abundant mucin invading with an acinar pattern. Histopathologically, the pulmonary masses in the present case were composed of abnormal papillary structures lined by single to double columnar neoplastic cells and forming arborized fronds supported by sub-epithelial fibrovascular stroma. Based on the characteristic histologic features, especially the pseudostratified appearance with taller columnar cell linings, the bovine pulmonary masses in the present case were confirmed as pulmonary papillary adenocarcinoma.

According to the manufacturer, the pan-CK AE1/AE3 antibody labels epithelial tissues from simple glandular to stratified squamous epithelium, whereas CK 7 labels PA derived from bronchial or bronchiolar epithelia but not squamous cell carcinoma. In addition, the CK MMF 116 antibody particularly labels bronchial epithelium and basal cells in stratified squamous epithelium. Chromogranin A and synaptophysin are useful markers for neuroendocrine tumors [8]. Therefore, the immunohistochemical profile obtained in this case, that is, pan-CK and CK 7 strongly positive, CK MNF116 positive sporadically, and chromogranin A negative, indicates that the pulmonary masses in the lungs of this case were of bronchial origin.

PAs in cattle should be differentiated from mesothelioma, other metastatic tumors of an epithelial origin, and granulo- matous inflammation caused by Mycobacterium spp. Gross lesions of primary pulmonary tumors vary from a single solid mass to multiple masses of variable sizes [8]. Advanced malignant tumors may produce smaller foci or nodules due to the invasion and the metastatic events of neoplastic cells. Metastatic lesions are more uniform in their size and distribution in the lung. Neoplastic cells in mesothelioma are both positive for epithelial CK and vimentin, a mesenchymal marker. Variably sized masses in pulmonary parenchyma and a negative result for vimentin suggested that the pulmonary masses in the present case are of primary lung tumor origin. Bovine uterine adenocarcinoma has a high ability to metastasize to parenchymal organs such as the lung and liver [10], but neoplastic masses of the uterus were not observed in this case. Presence of bovine tuberculosis was also successfully ruled out based on the negative result for $\mathrm{ZN}$ staining.

In humans, the common complications associated with pulmonary fungal or mycobacterial infections in patients with pulmonary neoplasia have been described $[11,12]$. Recently, there were several reports of PA with fungal infections that can mimic or coexist with pulmonary neoplasm in dogs [9]. However, there was no evidence of fungal or mycobacterial infections in this case.

To our best knowledge, this is the first report of PA in Hanwoo in Korea. Although bovine PA is very uncommon, it should be included in the differential diagnosis of cattle with a pulmonary mass, especially in a slaughterhouse situation.

\section{Acknowledgments}

This research was supported by the 2019 scientific promotion program funded by Jeju National University.

\section{References}

1. Wilson DW, Dungworth DL. Tumors of the respiratory tract. In: Meuten DJ (ed.). Tumors in Domestic Animals. 4th ed. pp. 380-392, Iowa State Press, Ames, 2002.

2. Stünzi H, Head KW, Nielsen SW. Tumours of the lung. Bull World Health Organ 1974;50:9-19.

3. Anderson LJ, Sandison AT. Pulmonary tumours found in a British abattoir survey: primary carcinomas in cattle and secondary neoplasms in cattle, sheep and pigs. Br J Cancer 1968;22:47-57.

4. Anderson WA, Davis CL, Monlux AW. A survey of tumors occurring in cattle, sheep, and swine. Am J Vet Res 1956; 17:646-677.

5. Kang MS, Kwon HJ, Park MS, Youn HY, Han MG, Kim DY. Pulmonary adenocarcinoma with brain metastasis in a Miniature Pincher. Korean J Vet Res 2004;44:621-624.

6. Kim YS, Bhang DH, Kim MK, Seo KW, Joo MS, Park JH, Choi MC, Lee CW, Han HR, Hwang CY. Primary lung tumors in five dogs. J Vet Clin 2005;22:288-295.

7. Travis WD. Pathology of lung cancer. Clin Chest Med 2011; 32:669-692.

8. Wilson DW. Tumors of the respiratory tract. In: Meuten DJ (ed.). Tumors in Domestic Animals. 5th ed. pp. 480-498. 
Wiley-Blackwell, Ames, 2017.

9. Maniam R, Selvarajah GT, Mazlan M, Lung Than LT. Pulmonary papillary adenocarcinoma with Aspergillus versicolor infection in a dog. Med Mycol Case Rep 2017;19:25-29.

10. Agnew DW, MacLachlan NJ. Tumors of the genital systems. In: Meuten DJ (ed.). Tumors in Domestic Animals. 5th ed. pp. 689-722. Wiley-Blackwell, Ames, 2017.

11. Bodey G, Bueltmann B, Duguid W, Gibbs D, Hanak H,
Hotchi M, Mall G, Martino P, Meunier F, Milliken S, Naoe S, Okudaira M, Scevola D, van't Wout J. Fungal infections in cancer patients: an international autopsy survey. Eur J Clin Microbiol Infect Dis 1992;11:99-109.

12. Kobashi Y, Yoshida K, Miyashita N, Niki Y, Matsushima T. Pulmonary Mycobacterium avium disease with a solitary pulmonary nodule requiring differentiation from recurrence of pulmonary adenocarcinoma. Intern Med 2004;43:855-860. 